\title{
Imperfect melting pot - analysis of changes in diversity and segregation of US urban census tracts in the period of 1990-2010
}

\author{
Tomasz F. Stepinski ${ }^{\mathrm{a}}$, Anna Dmowska ${ }^{\mathrm{a}, \mathrm{b}}$ \\ ${ }^{a}$ Space Informatics Lab, Department of Geography, University of Cincinnati, Cincinnati, USA, OH 45221-0131, USA \\ ${ }^{b}$ Institute of Geoecology and Geoinformation, Adam Mickiewicz University, Poznan, Poland
}

\begin{abstract}
Analyzing racial distribution and its temporal change in American urban areas is an active area of research. Most attention focused on assessing levels of racial segregation at the spatial scale of a metropolitan area. In this paper, we present an analysis of 1990-2010 changes to racial diversity and segregation on a much smaller spatial scale of an urban census tract. To access time-standardized racial information at the tract and at the census block scales we use multiyear compatible high-resolution population grids. Indices of racial diversity and segregation are calculated for over 30,000 tracts pooled from 41 metropolitan areas. Statistical analysis of this dataset reveals that during the 1990-2010 period urban tracts increased their diversity in line with diversity increases of entire metro areas, but unlike metros, they also increased their levels of segregation. We hypothesize that an increased tendency for the residences of people of the same race to spatially aggregate on the tract scale is the result of individuals exercising preferences regarding their neighbors in reaction to the nationwide increase in diversity of the American population. The study also re-derives diversity and segregation indices from the first principles of the information theory, highlights the need to think about the issue of racial diversity/segregation in terms of spatial patterns, and uses one-person-per-dot maps to connect diversity/segregation indices to actual racial patterns.
\end{abstract}

Keywords:

Racial diversity, segregation, information theory, visualization

\section{Introduction}

In the United States, the urban population consists of multiple races and ethnicities. This is the result of the continuing immigration of different ethnoracial groups into the U.S. A notion of a melting pot - a metaphor for a fusion of nationalities, ethnicities, and cultures - is a part of the American ethos. One check on the validity of the melting pot metaphor is to analyze temporal changes to residential multiracial diversity and segregation using data published by the U.S. Census Bureau. There are already numerous studies reporting on the results of such analyzes (Iceland et al., 2002; Fischer, 2003; Fischer et al., 2004; Fasenfest et al., 2004; Farrell, 2008; Farrell and Lee, 2011; Parisi et al., 2011; Holloway et al., 2012; Wright et al., 2014; Lee et al., 2014; Fowler et al., 2016; Ellis et al., 2018; Bellman et al., 2018).

In these studies, the predominant method of assessing racial diversity and segregation was to calculate

${ }^{*}$ Corresponding author. Email address: stepintz@uc.edu the Theil's entropy and information theory index (Theil and Finizza, 1971; Theil, 1972; Reardon and Firebaugh, 2002) for metropolitan areas divided mostly into their constituent census tracts. Notable exceptions are the works of Holloway et al. (2012) and Wright et al. (2014) who used a diversity-dominant race classification of census tracts for their analysis. Comparing such assessments from two different censuses yielded an assessment of changes to racial diversity and segregation on the spatial scale of a metropolitan area. In contrast, our primary interest in this paper is in changes to racial diversity and segregation on the spatial scale of an individual census tract. The paper has also two secondary goals pertaining to a methodology of quantifying racial diversity and segregation and to visualization of such quantification.

We start by pointing out that it is conceptually advantageous to think about an urban racial fabric in terms of a spatial pattern formed by individual inhabitants. Fig. 1 shows such racial patterns in 1990 and 2010 for the Cook County in Illinois which includes the city of 


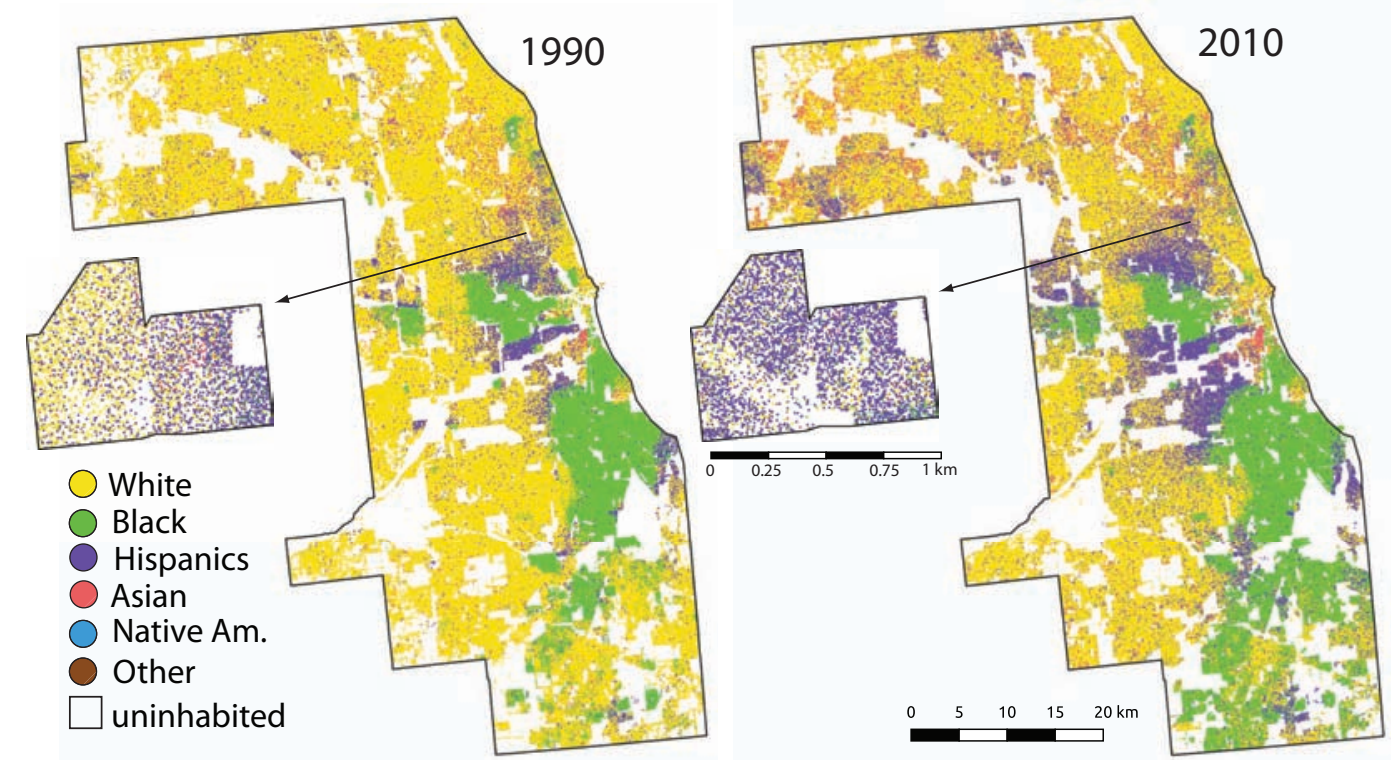

Figure 1: Racial patterns (dot maps) of the Cook County, Illinois in 1990 (left) and 2010 (right). Insets show racial patterns within a single tract.

Chicago. The racial patterns within a single tract located in the Cook County are also shown. These patterns were obtained using a cartographic method of racial dot maps (Roth, 2010) applied to high-resolution population grids (Dmowska et al., 2017). Each inhabitant of Cook County is depicted as a dot having a color corresponding to his race and placed in the best estimation of the location of his residence. Racial patterns provide an excellent visual tool. In racial patterns, gross shares of dots with different colors illustrate racial diversity. By visually analyzing the two maps it is clear that the diversity of the Cook County increased from 1990 to 2010 (a gross share of yellow dots decreased while gross shares of purple and red dots increased). The mixing of dots having different colors illustrates racial integration. Less mixing is observed in the 1990 map whereas more mixing is observed in the 2010 map indicating increasing racial integration (decreasing racial segregation) at the level of the entire Cook County.

We submit that to appreciate numerical assessments of changes in racial diversity and segregation, they need to be linked to corresponding racial patterns. Without such visuals, the change information cannot be connected to the "on the ground" reality. Thus, we developed a comprehensive set of exemplar patterns corresponding to all feasible types of racial patterns on the spatial scale of a census tract. This helps to translate racial change expressed in terms of numerical differences between indices calculated at different years into change expressed in terms of time modification of actual racial patterns.

Our second ancillary goal is a derivation of indices characterizing a racial pattern from the first principles of the Information Theory (IT). The IT, as originally formulated by Shannon (1948) was a theory of communication, but presently, the IT is also broadly used to quantify complexity and uncertainty. Given that racial patterns display complexity (see Fig. 1), and given the uncertainty in population data introduced by census areal aggregation, the IT is an ideal methodology to quantify racial pattern. In Section 2, we use fundamental concepts of the IT to frame the problem of quantifying racial patterns in terms of analyzing a joint probability distribution of race and location for the tract's inhabitants. This leads to a derivation of two indices - the entropy of the race variable (which assesses diversity) and the mutual information between the race and location variables (which assesses segregation). Derived indices turn out to be similar to the Theil's entropy and the Theil's information theory index commonly used in the racial segregation literature. We discuss discrepancies between the mutual information index and the Theil's information theory index and argue that the mutual information index is better suited to assess a level of racial segregation.

Finally, our main goal is to calculate 1990 and 2010 values of diversity and segregation indices for every one of over 30,000 tracts in 41 major metropolitan areas and statistically analyze 1990-2010 changes to these values. Our most important finding is that, statistically, the 
racial integration of urban tracts has decreased during the 1990-2010 period, even while their racial diversity has statistically increased. An analogous analysis at the county scale shows that, statistically, the racial integration of counties has not changed during the same period, while their racial diversity changed at the same rate as at the tract's scale. Thus, the melting pot hypothesis seems to break down on the smallest scale of individual neighborhoods. We provide a broadly-sketched hypothesis of social drivers that may explain these findings.

\section{Method: The Information Theory Approach}

Consider a hypothetical tract (Fig. 2) populated by 240 people of four different races (denoted by letters W, $\mathrm{B}, \mathrm{H}$, and $\mathrm{A}$ ) and subdivided into three blocks (denoted by symbols $b_{1}, b_{2}$, and $b_{3}$ ). Each person in this tract is characterized by two discrete random variables, $X$ his race, and $Y$ - a location of his residence (a block to which he is assigned). These variables are random because, due to an uncertainty introduced by census aggregation, we know only probabilities of each person's race and location. Let's denote by $p(X, Y)$ a joint probability distribution of race and location for a person randomly drawn from a tract. This joint probability distribution can be calculated directly from block-level census data by means of constructing a contingency table (a 2-D histogram of people in a tract) as shown in Fig. 2C. The distribution $p(X, Y)$ contains all information we can obtain from the census data about the spatial racial pattern of the tract. The pattern can be inferred from the values of $p(X, Y)$, but because a real tract consists of a large number of blocks, a direct inference of pattern from $p(X, Y)$ is not practical. Thus, we compress the information in $p(X, Y)$ into just two indices, one pertaining to compositional (thematic) complexity of the pattern and another pertaining to configurational (spatial) complexity of the pattern. Because the IT deals with extraction of information from a probability distribution function, it is ideally suited for the task of obtained these two indices.

\subsection{Information in tract-level data}

When using only a tract-level data there is no information about inhabitant's location (there are no blocks to consider) but there is information about inhabitant's race. This information is provided by a distribution $p(X)$, which can be obtained from $p(X, Y)$ by summing the values in each column of the contingency table (see Fig. 2C). For our exemplar tract $p(X)$ has the following values: $0.25(\mathrm{~W}), 0.5(\mathrm{~B}), 0.125(\mathrm{H})$, and $0.125(\mathrm{~A})$. Fig. 2A is a dot map depicting the exemplar tract - dots are randomly placed within a tract and assigned colors in accordance with $p(X)$. Tract-level data carries information about compositional complexity of tract's population but carries no information about its spatial complexity. Thus, an index that describes a tract's compositional complexity can be based only on $p(X)$. Such index is an entropy of $p(X)$ denoted by $H(X)$,

$$
H(X)=-\sum_{i=1}^{K} p\left(X=c_{i}\right) \log _{2} p\left(X=c_{i}\right),
$$

where $c_{i}, i=1, \ldots K$ are the labels of $K$ racial groups present in the tract, and $p\left(X=c_{i}\right)$ is a probability of a random person to belong to a racial group $c_{i}$ (also a relative share of $c_{i}$ subpopulation). Note that, in accordance with the IT convention, we use a symbol $H$ to denote entropy and $\log _{2}$ to calculate it. $H(X)$ is best interpreted as a similarity between the actual histogram of races in the tract $p(X)$ and the uniform histogram $u(X)$ (each race has an equal share which represents an ultimate diversity). Such interpretation follows from algebraic transformations resulting in expressing $H(X)$ in terms of the Kullback-Leibler (KL) divergence $d(p, u)$ (Chodrow, 2017a),

$$
H(X)=\log _{2} K-d(p, u)
$$

The $d(p, u)$ is a measures of a "distance" between histograms $p(X)$ and $u(X)$, it equals to zero if $p=u$ and it equals to $\log _{2} K$ if $p(X)$ has only a single bin (the tract is racially homogeneous). Thus, $H(X)$ is a measure of "similarity" between histograms $p(X)$ and $u(X)$, it equals to zero if histograms are maximally dissimilar (a tract is racially homogeneous) and it has a maximum value of $\log _{2} K$ if the tract has the uniform histogram. From the above description, it is clear that $H(X)$ is a measure of compositional complexity of racial pattern, or, in other words, a measure of racial diversity. For the exemplar tract $K=4$ and $H(X)=1.75$.

Note the connection between diversity and uncertainty. Assume that census would withhold information about racial shares in a tract leaving the total population count as the only information available. In the absence of any explicit or implicit knowledge about the race distribution, it is reasonable to assume a uniform histogram which reflects our maximum uncertainty about a race of a random tract's inhabitant. Once the actual shares are revealed our uncertainty decreases. The $H(X)$ quantifies the remaining uncertainty; if an actual histogram has only one bin, all uncertainty is removed, otherwise, some uncertainty remains. The racial diversity of the tract, as measured by $H(X)$, is an uncertainty as to the 

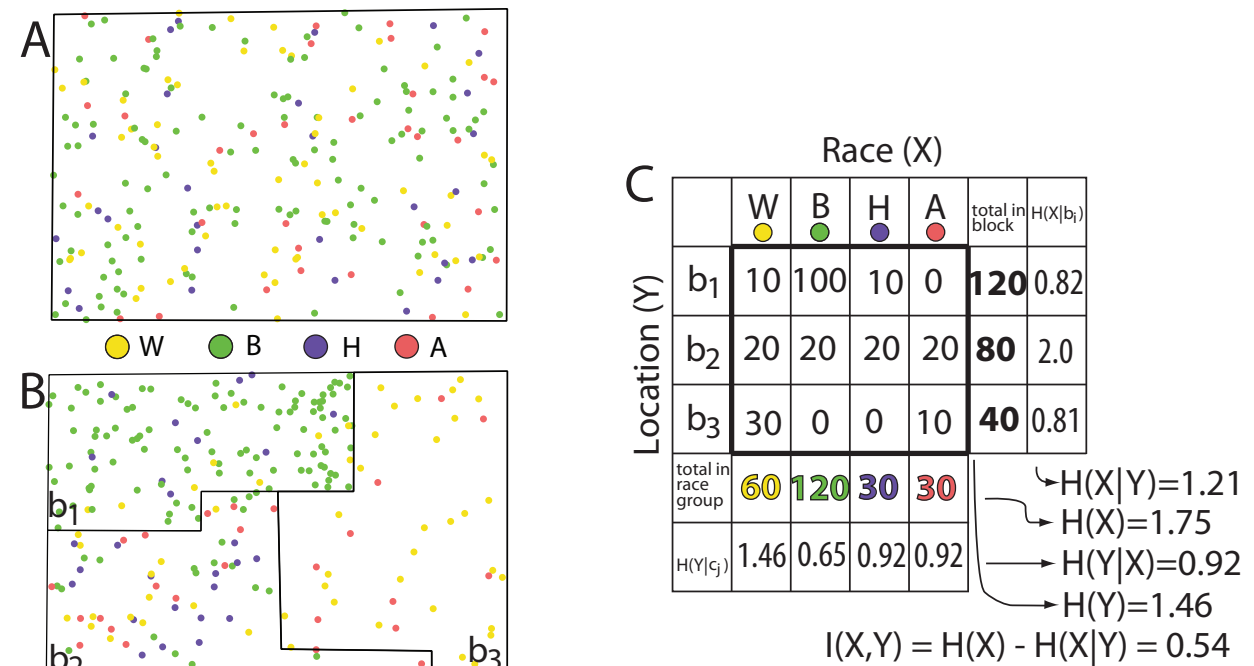

Figure 2: Hypothetical tract with 240 inhabitants subdivided into three blocks. (A) Racial dot map drawn on the basis of tract-level data. (B) Racial dot map drawn on the basis of block-level data. (C) A contingency table (within a heavily outlined rectangle) showing frequency distribution of people in the tract with respect of their race and block of residency. The next to the last column gives a block population count, the last column gives a value of diversity metric for each block. The next to the last row gives a race group count in the entire tract, the last row gives a value of uncertainty of finding a member of a given racial group in a specific block. See the main text for additional explanations.

race of its randomly selected inhabitant. In our exemplar tract the information contained in the tract-level data reduces the uncertainty about the race of a random inhabitant in the exemplar tract by $12.5 \%$ with respect to no information at all.

\subsection{Information in block-level data}

Using block-level data provides information about both, race and location of tract's random inhabitant. Information about inhabitant's race is provided by $p(X)$ and is not different from that provided by the tractlevel data. Information about inhabitant's location is provided by a distribution $p(Y)$, which can be obtained from $p(X, Y)$ by summing the values in each row of the contingency table (see Fig. 2C). For our exemplar tract $p(Y)$ has the following values: $0.5\left(\mathrm{~b}_{1}\right), 0.333\left(\mathrm{~b}_{2}\right)$, and $0.167\left(\mathrm{~b}_{3}\right)$. Note that $p(X, Y) \neq p(X) p(Y)$ which indicates that variables $X$ and $Y$ are dependent.

Fig. 2B is a dot map depicting the exemplar tract using the block-level data. Although the placement of dots within each block is random, it is not random with respect to the tract as a whole, in fact, we can observe some degree of correspondence between race and location (block). Tract's segregation increases (its racial pattern become less complex) with the increase of correspondence between blocks and races. In other words, the tract is increasingly segregated when we are (on average) increasingly less uncertain about a race of a per- son drawn randomly from a given block instead of from the entire tract.

The last column in a table shown in Fig. $2 \mathrm{C}$ gives such uncertainties when drawing from blocks $b_{1}, b_{2}$, and $b_{3}$ in the exemplar tract. Uncertainties associated with blocks $b_{1}$ and $b_{3}$ are indeed smaller than an uncertainty associated with the tract as a whole (1.75), but an uncertainty associated with block $b_{2}$ is larger. However, the population-weighted average of these three uncertainties $(H(X \mid Y)=1.21)$ is smaller than ancertainty associated with the tract as a whole (1.75). In fact, Jensen's inequality (Jensen, 1906) requires that $H(X)-H(X \mid Y) \geq 0$ for any tract, or any other region with subdivisions which are mutually exclusive and exhaustive. The difference between an uncertainty calculated from a tract and an average uncertainty calculated from its blocks,

$$
I(X, Y)=H(X)-H(X \mid Y),
$$

quantifies a degree of dependence between race and location variables and thus it quantifies a degree of tract's segregation. Thus, $I(X, Y)$ is our second index which pertains to configurational complexity of racial pattern. In the IT the metric $I(X, Y)$ is called mutual information. By virtue of Jensen's inequality $I(X, Y) \geq 0$; it is equal to 0 only if every block has the same shares of races as the entire tract (a racial integration of the tract is perfect), and it achieves a maximum value equal to $H(X)$ 

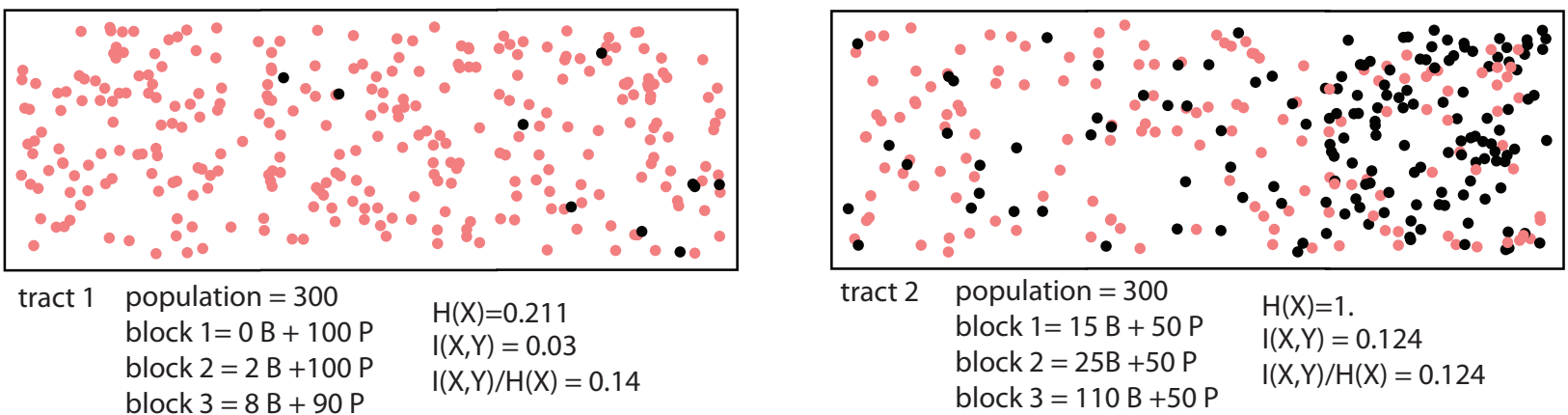

Figure 3: Two synthetic tracts constructed to have comparable values of the Theil's information theory index $\mathcal{H}$ despite their segregations levels being perceived as very different.

if every block is racially homogeneous. For the exemplar tract $I(X, Y)=0.54$. Thus, information contained in the block-level data reduces the uncertainty about the race of random inhabitant in the exemplar tract by $31 \%$ with respect to the information contained in the tractlevel data, or by $40 \%$ with respect to no information at all.

To summarize, using the IT we have derived two indices, $H(X)$ and $I(X, Y)$, which together provide a succinct description of tract's racial pattern. Functionally, the $H(X)$ is equivalent to not-normalized Theil's entropy and $I(X, Y)$ is equivalent to the not-normalized Theil's information theory index $\mathcal{H}$ (Reardon and Firebaugh, 2002). Note that $\mathcal{H}$ is a relative measure of segregation - in our context, a difference between diversity of the tract as a whole and a population-weighted average diversity of tract's constituent blocks expressed as a fraction of whole tract's diversity.

We submit that because of its relative construct, $\mathcal{H}$ is not best-suited to measure segregation. Consider two synthetic tracts visualized in Fig. 3. Both tracts, each divided into three blocks, have the same population of 300 people consisting of two racial groups denoted by B and P. The population mixes of these tracts are synthesized to be different; details are shown in Fig. 3. Visually, there is no doubt that tract 2 is more segregated than tract 1 . This is reflected by the value of $I(X, Y)_{2}$ being four times higher than the value of $I(X, Y)_{1}$. However, notice that $\mathcal{H}_{2}$ and $\mathcal{H}_{1}$ have comparable values with $\mathcal{H}_{1}$ actually slightly higher than $\mathcal{H}_{2}$. Thus, whereas $I(X, Y)$ correctly reflects observations, $\mathcal{H}$ does not. It is not that $\mathcal{H}$ is an incorrect measure of segregation, rather the relative segregation it measures is not the information that relates to how we perceive racial segregation (see also discussion in Frankel and Volij (2007)). An almost homogeneous tract (like the tract 1 in Fig. 3) is not perceived as racially segregated no matter how the mem- bers of a small minority are distributed. For a tract to be perceived as segregated it has to have a certain level of diversity. By measuring segregation relative to diversity $\mathcal{H}$ is missing this important insight. The problem is most conspicuous for low diversity tracts (which are very frequent in 1990 data), but it exists for all levels of diversity. Advantages of using $I(X, Y)$ over $\mathcal{H}$ for segregation studies was previously discussed by Frankel and Volij (2007) and Mora and Ruiz-Castillo (2008).

\section{Data and computations}

We analyze tracts pooled from 41 metropolitan areas (MSA). This dataset was previously used in Dmowska and Stepinski (2018) on the basis of two criteria: (1) the population of MSA was over 1 million in 2010, and (2) all major ethnoracial groups were present in 1990, 2000, and 2010. For our analysis, we need population counts in standardized tracts and blocks. A census block is the smallest geographic unit used by the U.S. Census Bureau for tabulation of 100-percent data; the median number of blocks in urban tracts is 59 and the median absolute deviation (MAD) is 27. Although standardized tracts are available, for example, from the National Historical Geographic Information System (NHGIS) (https://www.nhgis.org/) or from GeoLytics (http://www.geolytics.com/), standardized blocks are not available. Therefore, we use the SocScape (http://sil.uc.edu) high-resolution grids (Dmowska et al., 2017) which are available for 1990, 2000, and 2010.

SocScape grids are the results of dasymetic modeling (Dmowska and Stepinski, 2018); their values are designed to add exactly to corresponding census counts within each block and tract. Thus, for our purpose, the 2010 SocScape data is identical to the 2010 Census data. For 1990, we aggregate 1990 SocScape grid 

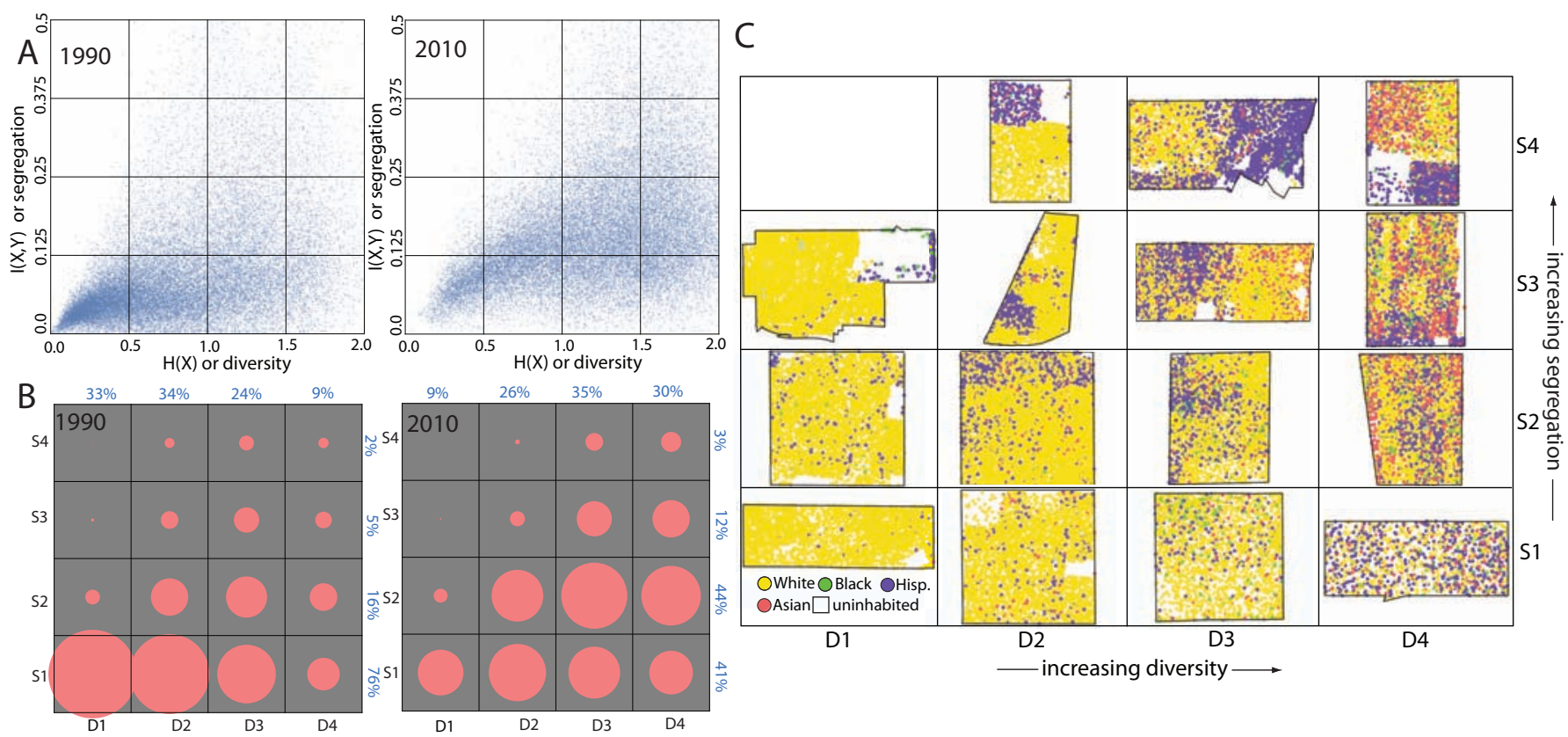

Figure 4: (A) Diversity-segregation (DivSeg) graphs constructed from 33,154 tracts collected from all 41 cities in 1990 and 2010. Ranges of $H(X)$ and $I(X, Y)$ correspond to their limits in the dataset and not to theoretical ranges of these indices. (B) DivSeg histograms. Areas of red circles are proportional to the fractions of tracts in histogram bins; the sum of all bins equals to 1. Percentages shown in blue are shares of tracts in a given diversity class (horizontal axis) and a given segregation category (vertical axis). (C) Exemplars of a typical racial configuration for tracts in each sector as visualized by a racial dot map. See the main text for additional explanations.

to 2010 boundaries of blocks and tracts. Differences between population and racial subpopulations counts in our standardized 1990 tracts and 1990 NHGIS standardized tracts are on average only $1 \%$. Counts for our 1990 block counts cannot be independently checked due to lack of analogous data. We subdivided population into six ethnoracial groups (Whites, Blacks, Hispanics, Asian, Native Americans, and others). For shortness, we don't use a "non-Hispanic" designation in front of Whites, Blacks, Asian, and Native Americans.

There are 33,154 tracts in our dataset. For each tract we calculate the values of $H(x)$ and $I(X, Y)$ in $t_{1}=1990$ and $t_{2}=2010$. Plotting the pairs of indices $\{H(X), I(X, Y)\}$ collected from all tracts in a given year as points in 2-dimensional graph results in the diversitysegregation (DivSeg) diagram depicting different patterns of racial distribution within urban tracts. Comparing DivSeg plots constructed for $t_{1}$ and $t_{2}$ reveals the tendencies of change in tracts' racial patterns (see Fig 4A.)

To simplify the analysis and to provide a visualization of those tendencies we classify all tracts into sixteen racial configuration types based on their values of $H(x)$ and $I(X, Y)$. To classify tracts we divided the DivSeg diagram into sixteen rectangular sectors. Tracts characterized by values of $H(x)$ and $I(X, Y)$ located within the same sector have similar values of diversity and segregation and thus belong to the same DivSeg class. Each DivSeg class is labeled by a combination of symbols, from D1 to D4, in the increasing level of diversity (low, enhanced, moderate, and high), and from $\mathrm{S} 1$ to $\mathrm{S} 4$, in the increasing level of segregation (low, enhanced, moderate, and high). Thus, for example, a racial configuration in a tract belonging to the class D4-S1 can be succinctly described as integrated diversity, but a racial configuration in a D4-S4 tract can be described as segregated diversity.

The sixteen classes encapsulate all possible configurations of racial patterns. Fig. 4C shows the division of DivSeg diagram into the sixteen sectors; an exemplar tract for each DivSeg class is given (no example of class D1-S4 was found among 33,154 tracts). Only exemplars in segregation classes S3 and S4 conform to the usual perception of what does it mean for the population to be segregated, configurations in exemplars of segregation class S2 can be better described as clustering or clumping. Thus, the term "segregation" commonly used to describe the meaning of the $I(X, Y)$ index may not be 
A. Tracts subdivided into blocks
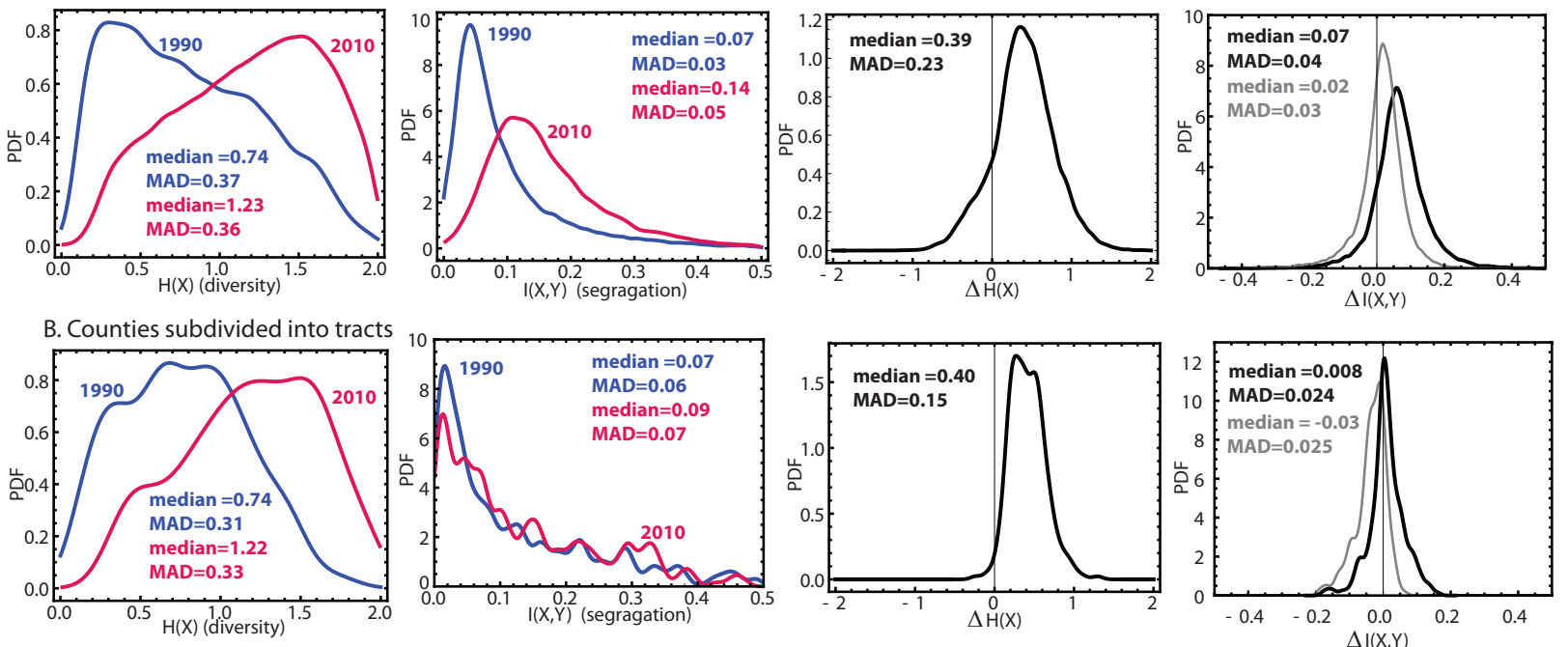

Figure 5: Kernel density estimations of distributions of the values of $H(X), I(X, Y), \Delta H(X)$, and $\Delta I(X, Y)$ from tracts divided into blocks (A) and counties divided into tracts (B). A wiggly character of $I(X, Y)$ distributions for counties is due to a small value of kernel bandwidth needed to assure that estimated distributions integrate to 1. Gray-colored distributions are for 1990-2010 change of the Theil's information theory index.

the best choice; the term "aggregation" would probably better reflect the meaning of this index. Note that, for visualization purposes, exemplars in Fig. 4 has been purposefully selected so the largest subpopulation is of the same racial group in each class, but the classes pertain to configuration and not to which racial group is dominant. Thus, a tract will maintain its class designation if its racial subpopulations are changed (colors of the dot on Fig. 4 are changed) providing that the pattern remains the same.

By counting tracts belonging to each class in a given year, we obtain DivSeg histograms with sixteen bins (Fig. 4B). We compare DivSeg histograms constructed from 1990 and 2010 data to reveal temporal trends in tracts' diversity and segregation levels.

\section{Results}

Fig. 5A shows distributions of the values of $H(X)$, $I(X, Y), \Delta H(X)$, and $\Delta I(X, Y)$ for urban tracts in our dataset; $\Delta$ indicates change, a difference between a 2010 value and a 1990 value of an index. Distributions of both indices have changed significantly between 1990 and 2010. In 1990 most likely values of $H(X)$ were low (population in most tracts was almost mono-racial), but in 2010 most likely values of $H(X)$ were high (population in most tracts was multi-racial). Thus, the population of U.S. urban tracts has become significantly more diverse during the $1990-2010$ period. The $I(X, Y)$ distribution has also shifted to larger values but by a relatively small amount. Thus, U.S. urban tracts had become somewhat more segregated during the 1990-2010 period. Note that, because of the shapes of the distributions, summary statistics, such as the mean and the standard deviation, or even the median and the median absolute deviation (MAD), are not very informative.

Perhaps the most important information is given by the two right-most panels in Fig. 5A which show distributions of 1990-2010 changes of $H(X), I(X, Y)$ values. In particular, statistically, the change of $I(X, Y)$ is positive - a majority of tracts had increased their segregation between 1990 and 2010. The distribution of changes in $\mathcal{H}$ is shown by a gray-colored curve. Statistically, tracts had also increased their values of $\mathcal{H}$.

For comparison, Fig. 5B shows distributions of the values of $H(X), I(X, Y), \Delta H(X)$, and $\Delta I(X, Y)$ for counties in our dataset. Our dataset consists of 273 counties; we divided these counties into standardized tracts for calculations of $I(X, Y)$. Comparing distributions of $H(X)$ for counties and tracts shows that, in 1990, counties were more likely to be diverse than tracts, but, in 2010, counties and tracts have similar probabilities of having a given level of diversity. Interestingly, distributions of county-level $I(X, Y)$ are almost identical in both years. This shows that segregation at the county scale (as assessed on the basis of their con- 


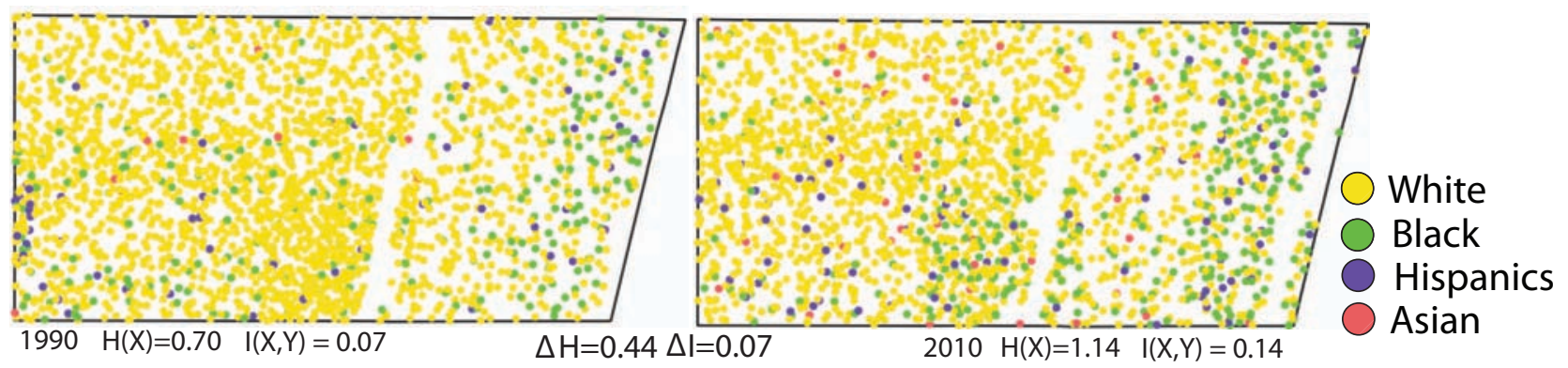

Figure 6: Dot maps of a tract which in 1990 had a typical (most likely) racial configuration, and which underwent a typical (most likely) $1990-2010$ change.

stituent tracts) remained statistically unchanged during the 1990-2010 period. In 1990, the county-level distribution of $I(X, Y)$ was very similar to the tract-level distribution of $I(X, Y)$, but while the tract-level distribution of $I(X, Y)$ has changed during the 1990-2010 period to reflect an increasing segregation at the tract level, the county-level distribution remained unchanged. However, note that if segregation is measured by $\mathcal{H}$, most counties decreased their segregation during the 1990-2010 period (see the right-most panel in Fig. 5B). This demonstrates that the conclusion about the trend of racial segregation may depend on whether segregation is assessed using an absolute measure $(I(X, Y))$ or a relative measure $(\mathcal{H})$.

Fig. 6 shows an example of a tract which in 1990 had a typical configuration and underwent a typical change during the 1990-2010 period. This visualizes what a typical increase of segregation by $\Delta I=0.07$ means for the change in the spatial pattern of racial subpopulations. In this example, the 1990-2010 change in the pattern is not large but it is clearly visible. Small increases in the tract's value of $I(X, Y)$ may be due to the random process instead of a systematic process (Leckie et al., 2012; Jones et al., 2018), however the fact that $85 \%$ of tracts have increased their values of $I(X, Y)$ points toward the existence of a systematic process.

Fig. 4B shows normalized DivSeg histograms for all tracts in our dataset constructed from 1990 and for 2010 data. Visually comparing the two histograms reveals that the overall tendency was for tracts to increase their levels of diversity and also to increase their levels of segregation in agreement with the results shown in Fig. 5A. The number of tracts in low/enhanced diversity classes (D1/D2) decreased from 33\%/34\% to $9 \% / 26 \%$, while the number of tracts in medium/high diversity classes (D3/D4) increased from $24 \% / 9 \%$ to $35 \% / 30 \%$. Similarly, the number of tracts in low/enhanced segregation classes $(\mathrm{S} 1 / \mathrm{S} 2)$ decreased from $76 \% / 16 \%$ to
$41 \% / 44 \%$, while the number of tracts in medium/high segregation classes (S3/S4) increased from $5 \% / 2 \%$ to $12 \% / 3 \%$.

Increased diversity of a tract's population increases the potential for an increased level of segregation because the maximum possible level of absolute segregation is higher for a more diverse population (see Eq.3). However, a higher level of diversity does not automatically translate into a higher level of segregation. In principle, there is nothing to prevent an increasingly diverse tract to maintain racial integration, that is, to evolve from, for example, class D1-S1 in 1990 to class D4$\mathrm{S} 1$ in 2010. However, the data shows that it is more likely that a tract classified as D1-S1 in 1990 evolved to a class D1-S2 to 2010, thus increasing its segregation while increasing its diversity.

DivSeg histograms show the initial (1990) and final (2010) statistics of tracts' racial configurations but not the details of how the change has occurred. During the 1990-2010 period, all tracts changed their $\{H(X), I(X, Y)\}$ values. If the change resulted in the reclassification of the tract on the DivSeg diagram we call it a transition; $80 \%$ of tracts in our dataset transitioned during 1990-2010, while 20\% did not change enough to be reclassified. A full accounting of transitions is provided by a $16 \times 16$ transition matrix. A $\{i, j\}$ entry in the transition matrix is a number of tracts which are classified as a class $i$ in 1990 and as a class $j$ in 2010, where $i$ and $j$ takes values \{D1 S1, D1 S2, ., D4 S3, D4 S4\}. Transition matrix shows statistics of types of changes and helps to understand the character of the process responsible for that change.

Fig. 7A is a visualization of the transition matrix. The transition matrix has the same organizations as the DivSeg histogram; it is divided into 16 sectors each corresponding to DivSeg classes in 1990. Tracts classified to a given class in 1990 are redistributed to other classes 

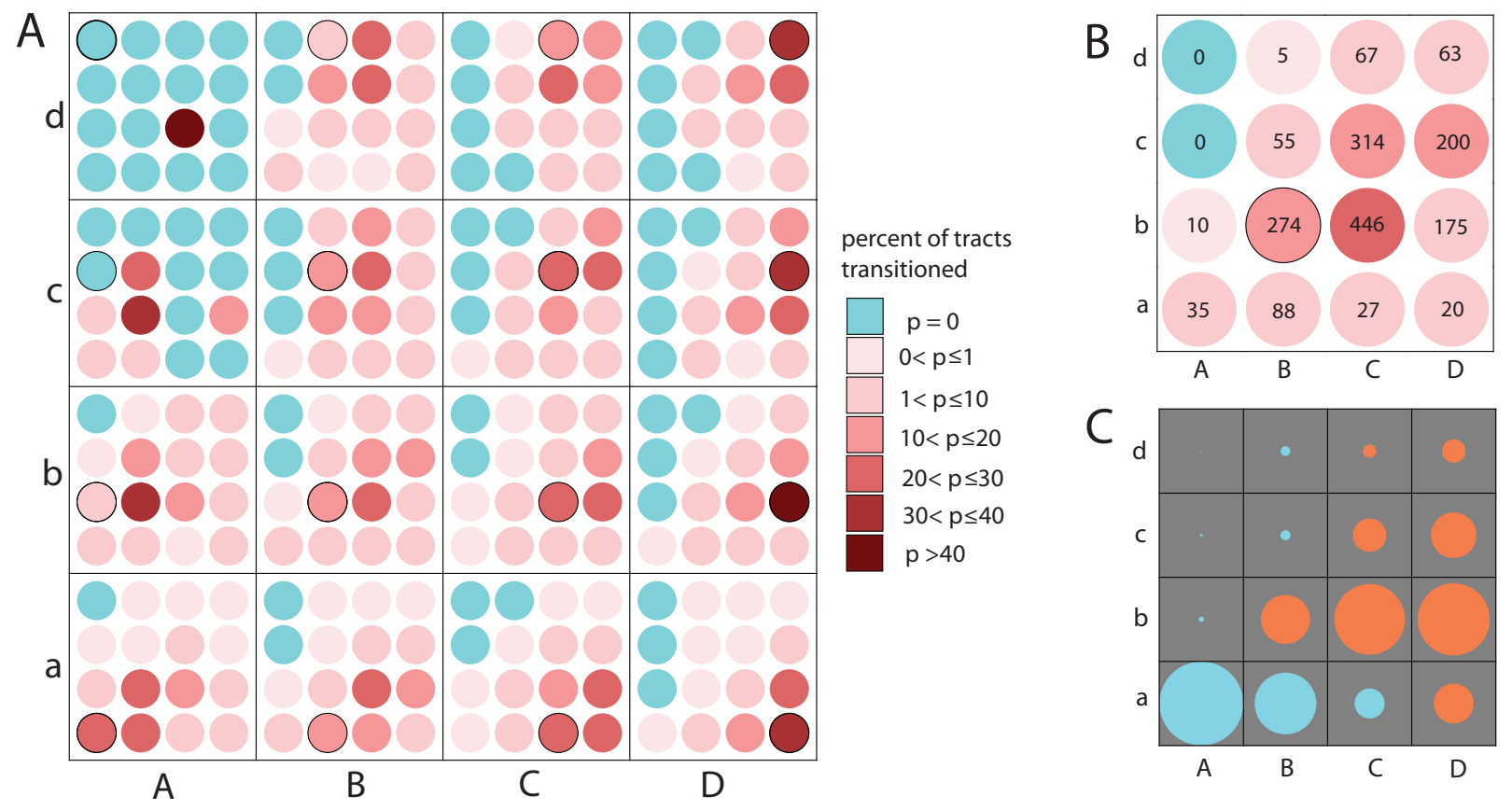

Figure 7: (A) Visualization of the transition matrix between racial configurational classes in 1990 and 2010 . Matrix is organized into sixteen $4 \times 4$ grids of circles. Each grid of circles show probabilities of transitions from a 1990 class to different classes in 2010 (see main text for details). Circles with black edges correspond to tracks not changing their class. (B) The Bb sector of transition matrix shown in more details. (C) Diagram illustrating a net change to DivSeg histogram bins during the 1990-2010 period. Areas of circles are proportional to the magnitude of the net change, an orange color indicates the net gain and blue color indicates the net loss.

due to the changes in their values of $H(X)$ and $I(X, Y)$ during the 1990-2010 period. This redistribution is depicted by a $4 \times 4$ grid of circles which correspond to $\mathrm{Di}-$ vSeg classes in 2010. A percentage of tracts transitioning from class $i$ in 1990 to class $j$ in 2010 is depicted by the color of a circle. Fig. 7B shows the D2-S2 sector of the transition matrix in greater details. In 1990 there were 1779 tracts classified as D2-S2 but only 274 of them maintained this classification in 2010. Remaining 1505 tracts had transitioned to other classes as indicated by numbers in Fig. 6B.

From Fig. 7A we observe a stochastic nature of tracts' evolution. Tracts having similar racial configurations in 1990 (belonging to the same class) evolve differently (there are many non-blue circles in each sector of transition matrix). This reflects local circumstances which vary from one location to another. If those circumstances were totally random, net gains and losses to histogram's bins membership in the DivSeg histogram will cancel themselves out and the histogram would not change between 1990-2010. However, the DivSeg histogram has changed (see Fig. 4) indicating the existence of prevailing trends in the transition matrix. The net change to bins membership in the DivSeg histogram is shown in Fig. 7C. In this figure blue circles indicate bins which lost tracts and orange circles indicate bins which gained tracts; the size of the circle indicate the magnitude of gain/loss. Most net loses are to the low/enhanced diversity, low segregation classes, while most net gains are to the moderate/high diversity, enhanced segregation classes. This reiterates our earlier conclusion that, statistically, tracts become more diverse and more segregated between 1990 and 2010.

Overall, our results show that the 1990-2010 change of racial patterns within U.S. urban tracts is toward more diversity (racial shares of tract's population become more even) but also toward more segregation (racial subpopulations within a tract tend to aggregate in different parts of the tract). Detailed social analysis of this observation is beyond the scope of this paper. Here we propose a following broadly-sketched hypothesis that may account for the results of our analysis. Increased diversity is due to nationwide social processes over which individuals have no direct control. This macro process permeates all spatial scales. However, social processes leading to racial aggregation at the tract scale are controlled by individuals. As diversities of tracts increase, individuals exercise their preferences 
regarding their neighbors (Schelling, 1971) which leads to an increasing racial aggregation (clumping of samerace households) throughout a tract. Because individual actions only redistribute populations within tracts but do not stop tracts' tendency to increase their diversity, a degree of the same-race aggregation at the county level remained the same or changed only slightly between 1990 and 2010.

\section{Discussion and Conclusions}

We analyzed levels of racial diversity and segregation within U.S. urban tracts and magnitudes of their changes during the 1990-2010 period. The most important result of our study is the finding that $85 \%$ of 1990 (standardized) tracts increased their segregation over the following 20 years, and $78 \%$ of tracts simultaneously increased their segregation and their diversity. We hypothesize that the tendency for the residences of people of the same race to spatially aggregate on a neighborhood scale is the result of individuals' reactions to nationwide societal changes coming to their neighborhood. If such a hypothesis is indeed correct, it indicates that, during the period of 1990-2010, there was some level of disconnect between the nationwide trend toward multiracial society and residential preferences of individuals. When the data from the 2020 census are released we would be able to check whether this trend continues.

In order to use the same, standardized tracts and blocks in 1990 and 2010, our analysis is performed using the gridded population data. Using gridded data does not influence the values of diversity and segregation in 2010, but, conceivably, it may lead to inaccurate population counts in 1990 and thus influence the values of segregation in that year. To check that this is not the case we performed two tests. (a) We use gridded data to calculate $\Delta \mathcal{H}$ at the county scale. The median value $\Delta \mathcal{H}$ is -0.03 (see Fig. 5), in broad agreement with $\Delta \mathcal{H}=-0.05$ as calculated from the 1990 and 2010 censuses for metropolitan areas (Lee et al., 2014), which are roughly of the same spatial scale as counties. This shows that gridded data and direct census data yield the same conclusions, at least at larger scales. (b) For the Cook County, Il we calculated values of $H(X)$ and $I(X, Y)$ directly from the 1990 census tracts and blocks data. Distributions of $H(X)$ and $I(X, Y)$ for 1990 tracts divided into 1990 blocks are almost identical to distributions of $H(X)$ and $I(X, Y)$ for standardized tracts divided into standardized blocks. These checks confirm feasibility of using SocScape grids to an analysis of diversity and segregation changes.
We stressed the importance of relating the values of diversity and segregation indices to racial patterns as visualized by dot maps. Racial patterns are as close to the "ground truth" of population distribution as we can get given the character of the data. In particular, a diversitysegregation grid ( Fig. 4C ) of exemplar racial patterns demonstrates that diversity and segregation are two fundamentally different aspects of racial patterns; diversity (increasing from left to right) pertains to ratios of shares of different races in a tract whereas segregation (increasing from bottom to top) pertains to the separation of different races into individual blocks.

These observations led us to classification of tracts into sixteen diversity-segregation (DivSeg) classes (Fig. 4B) and to the construction of the transition matrix (Fig. 7) which provided detailed information on how the membership of these classes changed between 1990 and 2010. The transition matrix revealed that a significant share of 1990 tracts changed their DivSeg classification to a variety of different classifications in 2010. This may be because we analyzed a pool of tracts from different metropolitan areas across the U.S. Future analysis will address possible regional differences in tract-level diversity/segregation changes, as well as possible differences in such changes between different parts of metropolitan areas (for example, city center vs. suburbia).

We used pairs (1990 and 2010) of racial patterns within the same tracts to visually assess a degree of pattern change. We compared such change assessment with the change assessment provided by numerical indices: $\Delta H(X)$ for the change in diversity, and $\Delta I(X, Y)$ or $\Delta \mathcal{H}$ for the change in segregation. We found that values of $\Delta H(X)$ and $\Delta I(X, Y)$ agree with our visual assessment, but values of $\Delta \mathcal{H}$ frequently disagree with our visual assessment.

The finding that values of $\Delta \mathcal{H}$ do not always correspond to what is seen in racial patterns is disconcerting because much of existing estimates of segregation change is based on $\mathcal{H}$. In Section 2 we show that $\mathcal{H}$ is $I(X, Y)$ relative to $H(X)$. Because of its relative design, $\mathcal{H}$ has desirable properties, its values are always in the $(0,1)$ range and the upper limit of $\mathcal{H}$ corresponds to a complete spatial separation of racial groups regardless of how many groups are present. However, this relative design of $\mathcal{H}$ has also unintended negative consequences. For tracts with a low level of diversity, the ratio $I(X, Y) / H(X)$ can be large because of the low value of the denominator, even if the value of the numerator is also low. This leads to a situation like the one illustrated in Fig. 3, when, if we assume that tract 2 is the tract 1 in the later time, $\Delta \mathcal{H}$ is negative and suggests decrease in segregation whereas visual inspection of patterns, as 
well as the value of $\Delta I(X, Y)$, suggests increase in segregation.

As recently discussed by Harris and Johnston (2018) there is no single, best measure of segregation, Different measures reflect different meanings of the term "segregation." In this paper, we proposed that different measures can be assessed, although only qualitatively, on the basis of the comparison of their values to the observed change of racial pattern. If we would use $\mathcal{H}$ instead of $I(X, Y)$ to measure tracts' segregation we would find that $66 \%$ of 1990 tracts increased their segregation, and $55 \%$ of tracts increased their segregation and their diversity. These probabilities are lower than those obtained using $I(X, Y)$, but the overall conclusion remains qualitatively the same - the majority of tracts increased their segregation during the 1990-2010 period. However, when we analyzed segregation of counties (divided into tracts), the choice of a segregation measure led to different conclusions, a no-change of the segregation level when using $I(X, Y)$ vs. a decrease of the segregation level when using $\mathcal{H}$ (see the counties segregation change distributions in Fig. 5). Therefore, it is important to scrutinize what a given measure of multiracial segregation means when interpreting the results of segregation change analysis.

Measures of multiracial segregation, the Thiel's entropy, and information theory index, had been widely used after they were postulated by Theil and Finizza (1971) and popularized by Reardon and Firebaugh (2002). However, it was recently noted (Chodrow, $2017 b$ ), that these indices sprang from the basic concepts of the IT. In Section 2 we presented a probabilistic formulation of the racial distribution problem and derived two indices which describe diversity and segregation directly from principles of IT. As the two derived indices happen to correspond to the indices already in use, the introduction of the probabilistic framework was not necessary to achieve the primary goal of this paper. However, we consider the introduction of this framework to be a valuable result in its own rights.

The probabilistic formulation provides a formal and complete framework for quantifying racial patterns. It can be extended to use with gridded data without any aggregated subdivisions (Nowosad and Stepinski, 2018). The quantification of the problem of racial distribution requires only the basic form of IT when one variable (race) provides information about the other (location). However, an extension of the basic form of IT (Williams and Beer, 2010) can be used to quantify how information from multiple variables (for example race and income) provides information about the location. Thus, the IT formulation can be extended to analyze corre- lations between multiple social factors and residential distribution of inhabitants.

Theil's information theory index is interpreted in terms of the Theil's entropy, but the Theil's entropy is not interpreted at all in population studies. Thus neither index has a convincing interpretation. The IT provides a clear interpretation of both indices. As shown in Section 2, $H(X)$ is an uncertainty as to the race of an inhabitant randomly selected from the tract and $I(X, Y)$ is an average reduction of this uncertainty if an inhabitant is randomly selected from a block within this tract. In the context of racial diversity and segregation, the term "uncertainty" has also a clear interpretation. Uncertainty is the expected number of yes/no optimally asked questions needed to determine a race of randomly drawn inhabitant. This interpretation is possible when the entropy is calculated using a logarithm with base 2 . Thus, to learn which one of the four possible races is a randomly selected inhabitant of the hypothetical tract featured in section 2, we need to ask on average 1.75 questions (average from the number of answers needed to learn the race of several inhabitants). However, when the inhabitant is drawn from any of the three blocks the average number of questions is only 1.21 .

Our goal in reintroducing indices of diversity and segregation in terms of IT is to take a fresh look at these well-established concepts. In this paper, by doing so, we reevaluated the Theils's information theory index vis-a-vis the mutual information, which is an older, and a broadly used index in multiple disciplines. Our results indicate that the issue of how to measure multiracial segregation is perhaps worth revisiting.

Acknowledgments. This work was supported by the University of Cincinnati Space Exploration Institute.

\section{References}

Bellman, B., Spielman, S. E., S., R., Franklin, 2018. Local Population Change and Variations in Racial Integration in the United States, 2000-2010. International regional science review 41(2), 233-255.

Chodrow, P. S., 2017a. Divergence, Entropy, Information: An Opinionated Introduction to Information Theory. arXiv preprint, 1708.07459.

Chodrow, P. S., 2017b. Structure and information in spatial segregation. Proceedings of the National Academy of Sciences 114(44), 11591-11596.

Dmowska, A., Stepinski, T. F., 2018. Spatial approach to analyzing dynamics of racial diversity in large US cities: 199020002010. Computers, Environment and Urban Systems 68, 89-96.

Dmowska, A., Stepinski, T. F., Netzel, P., 2017. Comprehensive framework for visualizing and analyzing spatio-temporal dynamics of racial diversity in the entire United States. PLoS ONE 12(3), e0174993. 
Ellis, M., Wright, R., Holloway, S., Fiorio, L., 2018. Remaking white residential segregation: metropolitan diversity and neighborhood change in the United States. Urban Geography 39(4), 519-545.

Farrell, C. R., 2008. Bifurcation, fragmentation or integration? The racial and geographical structure of US metropolitan segregation, 1990-2000. Urban Studies 45, 467-499.

Farrell, C. R., Lee, B. A., 2011. Racial diversity and change in metropolitan neighborhoods. Social Science Research 40 (4), 1108-1123.

Fasenfest, D., Booza, J., Metzger, K., 2004. Living Together : A New Look at Racial and Ethnic Integration in Metropolitan Neighborhoods, 1990-2000. Tech. rep.

Fischer, C. S., Stockmayer, G., Stiles, J., Hout, M., 2004. Distinguishing the geographic levels and social dimensions of US metropolitan segregation, 1960-2000. Demography 41(1), 37-59.

Fischer, M. J., 2003. The relative importance of income and race in determining residential outcomes in US urban areas, 1970-2000. Urban Affairs Review 38(5), 669-696.

Fowler, C. S., Lee, B. A., Matthews, S. A., 2016. The contributions of places to metropolitan ethnoracial diversity and segregation: Decomposing change across space and time. Demography 53(6), 1955-1977.

Frankel, D. M., Volij, O., 2007. Measuring segregation. Tech. rep., Economics Working Papers (20022016). 180. Iowa State University.

Harris, R., Johnston, R., 2018. Measuring and modelling segregation New concepts, new methods and new data. Environment and Planning B 45(6), 999-1002.

Holloway, S. R., Wright, R., Ellis, M., 2012. The Racially Fragmented City ? Neighborhood Racial Segregation and Diversity Jointly Considered. The Professional Geographer 64, 63-82.

Iceland, J., Weinberg, D., Steinmetz, E., 2002. Racial and Ethnic Residential Segregation in the United States : 1980-2000. Tech. rep

Jensen, J. L., 1906. Sur les fonctions convexes et les inégalités entre les valeurs moyennes. Acta Mathematica 30(1), 175-193.

Jones, K., Manley, D., Johnston, R., Owen, D., 2018. Modelling residential segregation as unevenness and clustering: a multilevel modelling approach incorporating spatial dependence and tackling the MAUP. Environment and Planning B 45(6), 1122-1141.

Leckie, G., Pillinger, R., Jones, K., Goldstein, H., 2012. Multilevel modeling of social segregation. Journal of Educational and Behavioral Statistics 37(1), pp.3-30. 37(1), 3-30.

Lee, B. A., Iceland, J., Farrell, C. R., 2014. Is ethnoracial residential integration on the rise? Evidence from metropolitan and micropolitan America since 1980. In: Diversity and disparities: America enters a new century . Russell Sage Foundation, pp. 415-456.

Mora, R., Ruiz-Castillo, J., 2008. A defence of an entropy based index of multigroup segregation. Tech. rep., Working Paper 07-76 Economics Series 45 Departamento de Economia, Universidad Carlos III de Madrid.

Nowosad, J., Stepinski, T., 2018. Information-theoretical approach to measuring landscape complexity. bioRxiv preprint, 383281 .

Parisi, D., Lichter, D. T., Taquino, M. C., 2011. Multi-scale residential segregation: Black exceptionalism and America's changing color line. Social Forces 89(3), 829-852.

Reardon, S. F., Firebaugh, G., 2002. Measures of Multigroup Segregation. Sociological Methodology 32(1), 33-67.

Roth, R. E., 2010. Dot Density Maps. In: Warf, B. (Ed.), The Encyclopedia of Geography. Thousand Oaks, CA, Sage, pp. 787-790.

Schelling, T. C., 1971. Dynamic models of segregation. Journal of Mathematical Sociology 1, 143-186.

Shannon, C. E., 1948. A mathematical theory of communication. Bell system technical journal 27(3), 379-423.

Theil, H., 1972. Statistical Decomposition Analysis. Amsterdam: North.
Theil, H., Finizza, A. J., 1971. A Note on the Measurement of Racial Integration of Schools by Means of Informational Concepts. The Journal of Mathematical Sociology 1(2), 187-193.

Williams, P. L., Beer, R. D., 2010. Nonnegative decomposition of multivariate information. arXiv preprint, 1004.2515.

Wright, R., Ellis, M., Holloway, S. R., Wong, S., 2014. Patterns of racial diversity and segregation in the United States: 1990-2010. The Professional Geographer 66(2), 173-182. 